\title{
Aspergillus fumigates spondylodiscitis in an immunocompetent patient
}

\begin{abstract}
Spondylodiscitis is an inflammatory disease of one or more vertebral bodies and of corresponding intervertebral disc, the fungal etiology is rare, affecting mostly immunocompromised individuals. It is often delayed diagnosis by the indolence of symptoms, presenting itself as serious infection may result in important functional consequences. Here is a 69 year old lady presented with complaints of low back pain and bilateral lower limb weakness. History of hypertension and electrolyte imbalance present. Diagnosed as spondylodiscitis L2-L3 with MRI. Patient underwent discectomy and biopsy. Culture grew Aspergillus fumigatus and Aspergillus galactomannan test is positive. No evidence of immunocompromised status was found. Treatment was started with Variconazole.
\end{abstract}

Volume 7 Issue I - 2017

\section{Suresh S Pillai,' Sivasankar Reddy, ${ }^{2}$ Sujith} Thampy ${ }^{3}$

'Consultant Spine surgeon and HOD, Department of Orthopedics, India

${ }^{2}$ Resident in Orthopedics, Department of Orthopedics, India ${ }^{3}$ Consultant in Orthopedics, Department of Orthopedics, India

Correspondence: Suresh S Pillai, Consultant Spine surgeon and HOD, Department of Orthopedics, India,

Email sureshorth@googlemail.com

\section{Introduction}

Spondylodiscitis remains rare but its incidence rising, due to an increasingly susceptible population and the availability of more effective diagnostic tool. ${ }^{1}$ Fungal spondylodiscitis is relatively uncommon, mainly in non immuno compromised status. Diagnosis and treatment are challenging, requiring a multidisciplinary team. An illustrative case report and literature review presented here.

\section{Case report}

A 69yrs old lady presented to orthopedic outpatient department on $02 / 06 / 2016$ with complaints of back pain since 3 months duration. History of on and off fever for past 4months, decreased appetite, weight loss, decreased sleep, fatigue.

No history of trauma, bowel or bladder disturbance. She is a known case of hypertension, dyslipidemia and electrolyte imbalance.

\section{Physical examination}

pallor present and diffuse tenderness over mid back at D6,D7 region and D10,D12 region, straight leg rising test (SLRT) negative, bilateral ankle reflex exaggerated, Plantar flexor bilateral, No neurovascular deficit.

\section{Investigations}

Blood investigations-Total WBC count-13,300, ESR$111 \mathrm{~mm} / 1 \mathrm{sthr}$, CRP-58, ALP-215 IU, Albumin-3.8gm\%, Globulin$4.2 \mathrm{gm} \%$ (Table 1 ).

MRI showed evidence of spondylodiscitis with paraspinal and epidural inflammation at L2-L3 levels and altered signal changes at D5, D6,D7,D9,D10 \& D11 - suggestive of spondylitis (Figure 1).

A CT guided biopsy was attempted from the radiology department. But sample was in adequate for a diagnosis from the pathology department. So she advised open biopsy. Relatives refused it. Then she missed follow up and was on ayurvedic treatment for 2 months without any relief. She presented to the outpatient department again on $27 / 08 / 2016$, with complaints of weakness of both lower limbs more on the right side, Bowel habits - constipated, Bladder function normal. On examination she had diffuse tenderness in mid thoracic to upper lumbar region, SLRT negative.

\begin{tabular}{ll}
\hline Right side & Left side \\
\hline Hip flexor (LI L2) & Grade 2 Grade 4 \\
Knee extensor (L3) & Grade 2 Grade 4 \\
Ankle dorsi flexors (L4) & Grade 3 Grade5 \\
EHL (L5) & Grade 3 Grade5 \\
Ankle plantar flexion (SI) & Grade 5 Grade5
\end{tabular}

Retroviral markers-Negative, ECHO shows-no vegetations, Urine culture grew- Pseudomonasaurogenosa.

MRI Lumbo sacral Spine showed altered signal intensity involving L2-L3 vertebral endplates and intervertebral disc with enhancing pre and paraspinal soft tissue component. Extension noted into the posterior elements of L3 vertebral body with posterior epidural component. No significant canal stenosis noted at this level.

Table I Blood investigation

\begin{tabular}{llll}
\hline $\begin{array}{l}\text { Blood } \\
\text { paramete }\end{array}$ & $\mathbf{0 4 / 0 6 / 2 0 1 7}$ & $\mathbf{2 7 / 0 8 / 2 0 1 9}$ & $\mathbf{0 1 / 1 0 / 2 0 1 7}$ \\
\hline Counts & 13,300 & 16,700 & 20,600 \\
ESR & 111 & 122 & 116 \\
CRP & 58 & 113 & 72 \\
\hline
\end{tabular}

MRI Dorsal spine shows -Contiguous altered vertebral body signal intensity also imaged at D4 to D12 levels with enhancing pre and paraspinal soft tissue component. Epidural component noted at this level with e/o cord compression and cord signal changes, Disease shows progression when compared to MRI on 4/6/16 (Figure 2).

Stain, AFB Stain, TB-PCR, Culture and sensitivity (bacterial, fungal, anaerobic culture).

Investigations-Gram stain-Negative, AFB Stain-Negative, TBPCR-Negative Histopathology-Showed fibrocartilagenous tissue with 
collections of neutrophils and lymphocytes., Numerous branching slender septate hyphae are seen (Figure 3).

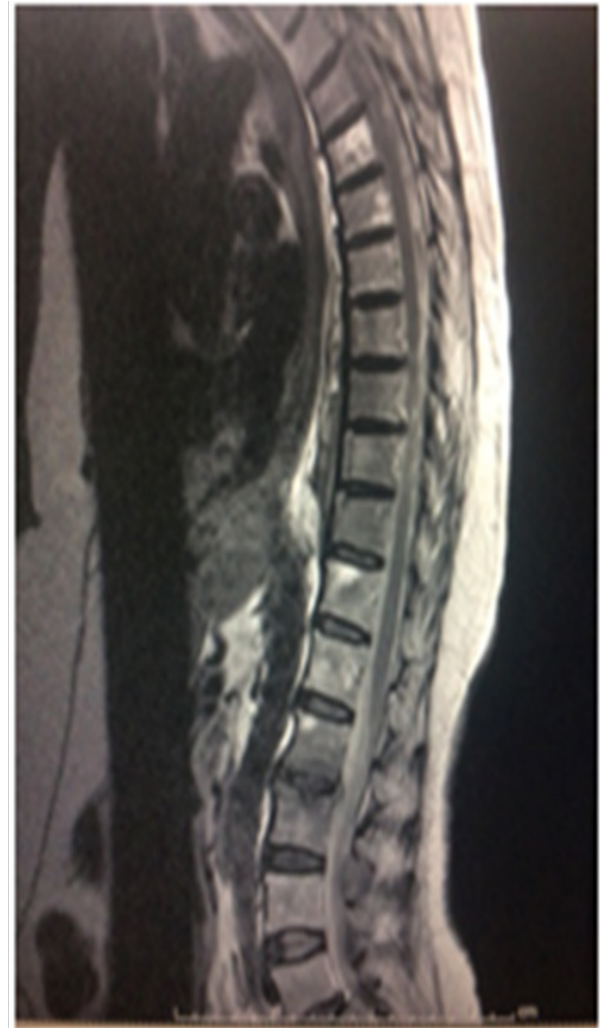

Figure I MRI on 04/06/2016.

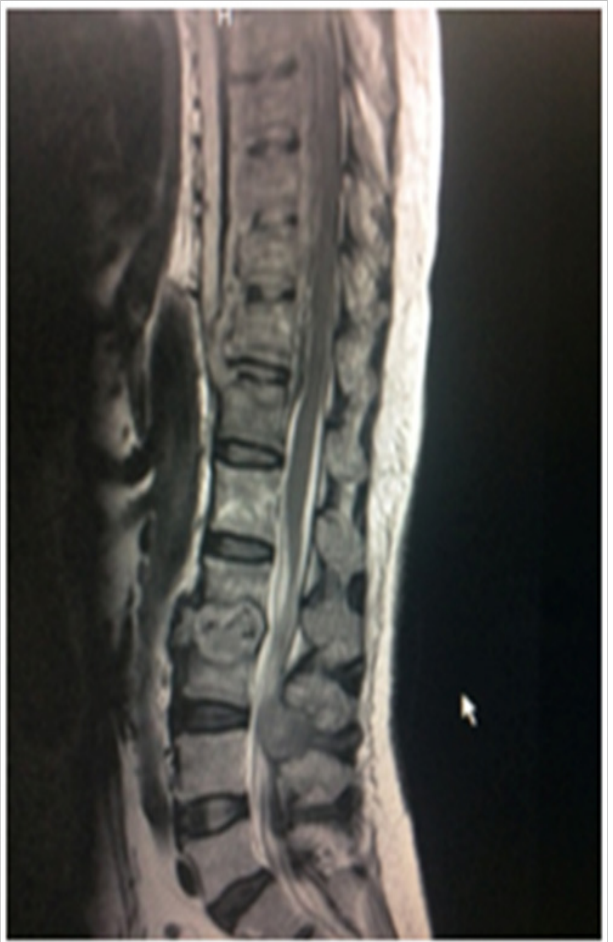

Figure 2 MRI was 27/08/2017.

Discectomy and biopsy L2-L3 was done on 30/08/2016, send for gram

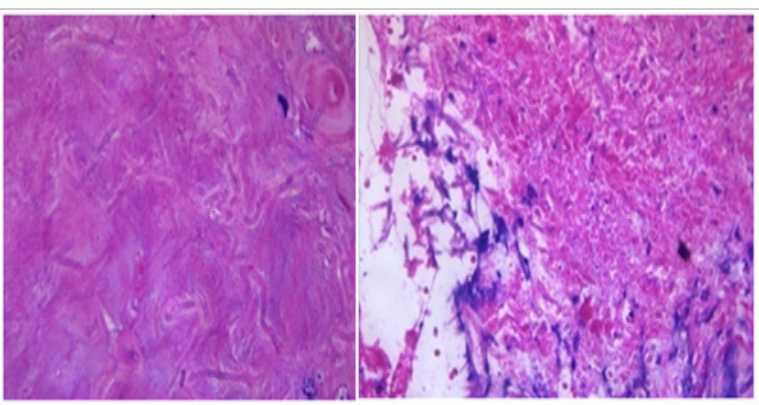

Figure 3 L2-L3 disectomy specimen shows Aspergillus fumigatus. Serum Galactomannan testis - 4.7>0.5 is positive.

\section{Treatment}

Initially ATT was started empirically along with pseudomonas sensitive antibiotics according to culture report after discussion with pathologist and microbiologist ATT Stopped and Antifungal Voriconazole $200 \mathrm{mg}$ started twice daily. She was responding to treatment. Her pain decreased, wound healed and no improvement in the neurology was noticed (Figure 4).

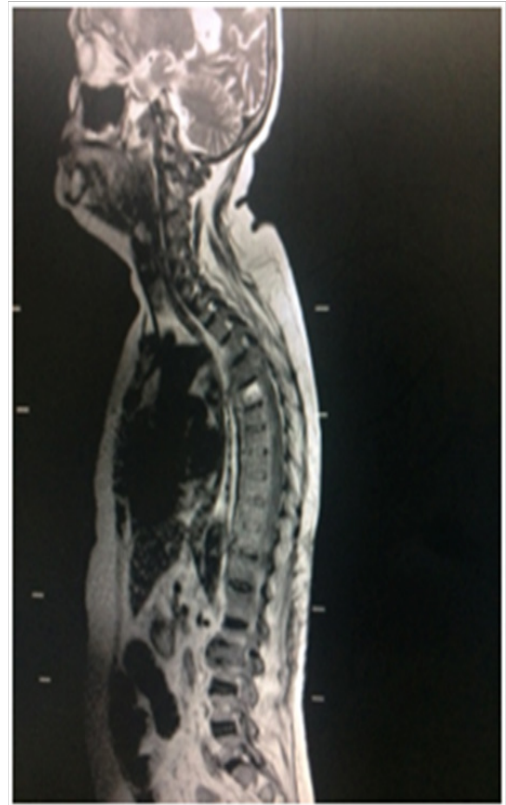

Figure $4 \mathrm{MRI}$ on $0 \mathrm{I} / \mathrm{I0} / 20 \mathrm{I}$.

\section{Outcome and follow-up}

She came for follow up at 6 weeks in the outpatient department symptomatically better and responding to treatment. ESR and CRP reduced marginally. Repeat blood culture showed no growth. MRI shows Multifocal involvement of lumbar and thoracic and intervertebral discs with evidence of canal stenosis and cord compression at thoracic level, no significant change compared to previous study, posterior paraspinal soft tissue with abscess formation on right side from D11-L3 level- a new development. The case was further discussed with microbiologist and infectious disease specialist at our hospital. They advised to continue the same treatment as she was symptomatically improving. But 1 month later the relatives informed us that the patient expired at home. 


\section{Discussion}

The term spondylodiscitis means primary infection of intervertebral disc by a pathogen, with secondary infection of neighbouring vertebral bodies $^{2}$ spondylodiscitis is a rare disease accounting for $2-7 \%$ of all cases of pyogenic osteomyelitis with incidence varying from 1 per 10000/year to 1per 250000/year, men affected 3times more than women. ${ }^{3}$

Any age group can be affected, although spondylodiscitis is most frequent in fifth to seventh decade of life, ${ }^{2}$ however incidence increasing due to longer life expectancy with chronic debilitating disease, immunosuppressive therapy, increasing use of indwelling devices and spinal surgery. ${ }^{3}$ Haematogenous spread of pathogenic micro organism into the vertebral bodies is most common source of infection in adults, it affects preferentially the lumbar spine followed by thoracic and cervical $(58 \%, 30 \%, 11 \%)$, possibly reflecting the relative proportion of blood flow. ${ }^{1}$ contagious infection is less common and is difficult to distinguish from direct inoculation, ${ }^{4}$ direct inoculation may occur during lumbar puncture, spinal anaesthesia, discography, chemonucleolysis, after spine surgery. ${ }^{4}$

Most important infecting organism in pyogenic osteomyelitis is staphylococcus aureus, accounting for more than $50 \%$ cases in most series from developed countries with a growing relevance of MRSA strains. ${ }^{5}$ However reported pathogens are large, less common but important pathogen include, enteric gram negative bacteria $39 \%$, streptococci $19 \%$, skeletal tuberculosis in $5 \%$ HIV negative patients and $60 \%$ in HIV positive patients. ${ }^{2}$ Brucellosis most common zoonosis in endemic areas, can account for $21-48 \%$ of spinal infections, representing the predominant cause in some series from Mediterranean basis and middle east.

Fungal infection of spine is relatively uncommon $0.5-1.6 \%$ usually. Vertebral aspergillos is can be classified in to 3 major categories, depending on the mode of acquisition. Direct inoculation related to trauma, spinal surgery, or epidural injection, contiguous spread from pleuro pulmonary disease which generally affects thoracic spine ${ }^{6}$ Haematogenous infection arising from pulmonary focus occur mainly in immuno supressed patients and is caused almost exclusively by Aspergillusfumigatus. Hematogenously acquired A. flavus and Aspergillusterreus, vertebral osteomyelitis has been associated with illicit intravenous drug use. Reported cases of vertebral aspergillosis in immunocompetent hosts were used almost exclusively by $A$. fumigatus. Most were acquired hematogenously. Although onequarter occurred following a spinal procedure. Inappropriate settings vertebral osteomyelitis secondary to aspergillos is need to be considered in immunocompetent patient with chronic back pain.

Back pain is the most common presenting symptom. Most patient developed discitis and osteomyelitis at 1 or multiple vertebral levels or epidural abscess, ${ }^{7}$ Early diagnosis of aspergillus spondylitis remain a challenge and require high degree of suspicion. Aspergillus spondylitis is confused with tuberculous spondylitis. Biopsy performed was definitive of diagnosis .Recently discovered biomarkers like aspergillus galactomannan, serum beta D-glucan can be used to confirm the diagnosis .Length of antifungal therapy for vertebral aspergillosis has not been established. IDSA guidelines suggest a minimum of 6-8weeks of antifungal therapy (Amphotericin $\mathrm{B}$ or Variconazole) in non -immunocompromised with aspergillus osteomyelitis, ${ }^{8}$ superiority of variconazole over amphotericin B for primary therapy of invasive aspergillosis has been demonstrated. Both itraconazole and variconazole are less toxic than amphotericin B. Most authors favour surgical treatment if there is advanced disease or neurological impairment, ${ }^{9}$ patients who received combined medical and surgical therapy had favourable outcome. ${ }^{10}$

\section{Learning points}

i. Fungal spondylodiscitis is a rare entity, especially in an immuno competent patient

ii. Serious threat to the patient's life due to diagnostic delay

iii. It remains a diagnostic and therapeutic challenge, which may result in important functional consequences.

iv. Patient's consent is essential for evaluation.

\section{Acknowledgements}

None.

\section{Conflict of interest}

The author declares no conflict of interest.

\section{References}

1. Katke RD. A huge benign mucinous cystadenoma of ovary: a case report and review of literature. Int J Reprod Contracept Obstet Gynaecol. 2014;3(2):456-459.

2. Gouliouris T, Aliyu SH, Brown NM. Spondylodiscitis: update on diagnosis and management. J AntimicrobChemother. 2010;65(3):11-24.

3. Sobottke R, Seifert H, Fatkenheuer G, et al. current diagnosis and treatment of spondylodiscitis. Dtsch Arztebl Int. 2008;105(10):181-187.

4. D’Agosto C, Scorzolini L, Massetti AP, et al. A seven-year prospective study of on spondylodiscitis: epidemiological and microbiological features. Infection. 2010;38(2):102-107.

5. BaleriauxDL, NeugroschlC. spine and spinal cord infection. EurRadiol. 2004;14:E72-E83.

6. Lew DP, Waldvogel FA. Osteomyelitis. Lancet. 2004;364(9431):369379 .

7. Lenzi J, Agrillo A, Santoro A, Marotta N, et al. Postoperativespondylodiscitis from aspergillus fumigatus in immunocompetent subjects. $J$ Neurosurgery sci. 2004;48(2):81-85.

8. Martinez M, Lee AS, Hellinger WC, et al. Vertebral osteomyelitis and acute diskitis in patient with chronic obstructive pulmonary disease. Mayo Clinic Proc. 1999;74(6):579-583.

9. Walsh TJ, Anaissie EJ, Denning DW, et al. Treatment of aspergillus: clinical practice guidelines of the infectious Diseases Society of America. Clin infect Dis. 2008;46(3):327-360.

10. Van Ooij A, Beckers Jm, Herpersmj, Et Al. Surgical Treatment Of Aspergillus Spondylodiscitis. Eur Spine J. 2000;9(1):75-79. 